\title{
PDTM: Poisson Distribution-based Trust Model for Web of Things
}

\author{
Weidong Fang \\ Science and Technology on \\ Micro-system Laboratory, Shanghai \\ Institute of Micro-system and \\ Information Technology, Chinese \\ Academy of Sciences, Shanghai \\ 201800, China.; University of Chinese \\ Academy of Sciences, Beijing 100049, \\ China \\ weidong.fang@mail.sim.ac.cn
}

\author{
Wuxiong Zhang \\ Science and Technology on \\ Micro-system Laboratory, Shanghai \\ Institute of Micro-system and \\ Information Technology, Chinese \\ Academy of Sciences, Shanghai \\ 201800, China \\ wuxiong.zhang@mail.sim.ac.cn
}

\author{
Wei Chen \\ School of Mechanical Electronic and \\ Information Engineering, China \\ University of Mining and Technology \\ (Beijing), Beijing 100083, China.; \\ School of Computer Science and \\ Technology, China University of \\ Mining and Technology, Xuzhou \\ 221116, China \\ chenw@cumt.edu.cn
}

\begin{abstract}
$\mathrm{Li} \mathrm{Yi}$
Science and Technology on

Micro-system Laboratory, Shanghai

Institute of Micro-system and

Information Technology, Chinese

Academy of Sciences, Shanghai

201800, China.; University of Chinese

Academy of Sciences, Beijing 100049,

China

969539394@qq.com
\end{abstract}

\begin{abstract}
Web of things (WoT) are inclined to suffer from internal attacks, which are from compromised nodes. Due to resource-constraint of WoT, the traditional security methods cannot be deployed. One of the most appropriate protection mechanisms to resist internal attacks is the trust management system. For the sake of evaluate the performance of WoT reasonably and appropriately, we improve the Beta-based reputation system, and propose a Poisson Distributionbased trust model (PDTM) in this paper. In view of evaluating a sensor node (or terminal) behaviors, its reputation and trust are represented by the Poisson distribution. PDTM is used to look for reliable nodes to transmit data and weaken malicious attacks within WoTs. The simulation results indicate that the PDTM can resist internal attack effectively, in order to strengthen the network security.
\end{abstract}

\section{KEYWORDS}

Web of things (WoT), Trust model, Poisson distribution, Internal attacks

This paper is published under the Creative Commons Attribution 4.0 International (CC-BY 4.0) license. Authors reserve their rights to disseminate the work on their personal and corporate Web sites with the appropriate attribution.

WWW'21 Companion, April 19-23, 2021, Ljubljana, Slovenia

(C) 2021 IW3C2 (International World Wide Web Conference Committee), published under Creative Commons CC-BY 4.0 License.

ACM ISBN 978-1-4503-8313-4/21/04

https://doi.org/10.1145/3442442.3451144
Weiwei Gao

Science and Technology on

Micro-system Laboratory, Shanghai

Institute of Micro-system and

Information Technology, Chinese

Academy of Sciences, Shanghai

201800, China

weiwei.gao@mail.sim.ac.cn

ACM Reference Format:

Weidong Fang, Wuxiong Zhang, Wei Chen, Li Yi, and Weiwei Gao. 2021. PDTM: Poisson Distribution-based Trust Model for Web of Things. In Companion Proceedings of the Web Conference 2021 (WWW'21 Companion), April 19-23, 2021, Ljubljana, Slovenia. ACM, New York, NY, USA, 5 pages. https://doi.org/10.1145/3442442.3451144

\section{INTRODUCTION}

As the name suggests, the web of things (WoT) means all things on the web page. Technically speaking, all things are connected to the web based on the REST protocol, which based on the HTTP protocol. In other words, WoT comes from the Internet of Things (IoT). It is a decentralized IoT. It also can make things linkable and discoverable by providing 'Things URL' on the network, and define standard data models and application programming interface (APIs) to make it interoperable. WoT is not just another vertical IoT technology stack that competes with existing platforms. It aims to serve as a unified horizontal application layer that bridges multiple potential IoT protocols together.

For WoT, it has data acquisition, processing and transmission capabilities, and can be deployed in many application fields [1-4]. Due to unattended and harsh deployment environment, as well as the openness of wireless media, sensor nodes are vulnerable to security attacks. Hence, their security threats are becoming increasingly prominent. In many applications, sensor nodes are easily captured and compromised by an adversary, in order to disclose the node's encryption key. The result is that the compromised node may be mistaken for a normal node in the network, and making the adversary to intercept, delete, insert, and tamper information 
is possible. It can be said that, once the nodes are compromised, the availability and integrity of the entire network is destroyed. Therefore, the cyber security is a critical issue that needs to be addressed.

In cyber security, the asymmetric cryptography is widely used to defend against those external attacks in the distributed network, which including Internet, Peer-to-Peer, and Ad Hoc networks. However, due to the complexity and huge computational memory, these encryption algorithms are not suitable for resource-constrained terminal. In addition, encryption-based security mechanisms can only address external security threat issues and cannot effectively defend against the internal attacks. This is due to that, the particularity of the node makes the WoT different from other networks. The node can refuse to cooperate with the service requester in order to save resources, namely, selfish node. Although they do not actively attack the network, a large number of selfish nodes maybe bring about serious consequences. The existing encryption mechanism does not distinguish the malicious nodes from the authenticated selfish nodes. Fortunately, some studies show that the trust management scheme is an effective approach to defend against the internal attacks [4]. The rest of this paper is organized as follows: shows the related trust management systems are shown in section 2 . In section 3, a Poisson distribution-based trust model is proposed in detail. In section 4, the simulation and analyses are provided. Finally, the conclusions are given in section 5 .

\section{RELATED WORKS}

Up to now, many trust models are proposed [14-20] to observe node behaviors and resist internal attacks from compromised nodes. Some typical works are discussed as follows.

Xia et al. [14] designed a trust management system, which combined multiple trust decision factors. Information entropy was used to quantify trust, which combined many kinds of trust factors to analyze the uncertainty and inhomogeneity of trust relationship from different angles. The fuzzy analytic hierarchy process was adopted to assign weight to each trust decision factor, more importantly, the fuzzy logic rule was used to predict and update the trust value for the next trust decision could be made.

Zhu et al. [15] presented an authenticated trust and reputation calculation and management (ATRCM). The method not only verified the authenticity of Cloud Service Providers (CSPs) and Sensor Network Providers (SNPs), but also determined the attribute relationship between a Cloud Service User (CSU) and a CSP through the service cost, trust and reputation of CSP and SNP. More importantly, it could effectively resist the main attacks in the trust management system, such as collusion attack and so on. Unfortunately, its algorithm complexity was large, which was a fatal problem for sensor networks with limited energy.

Wang et al. [16] incorporated data aggregation and communication failure into the trust model, and proposed a lightweight trust management system. The direct trust was evaluated by successful or unsuccessful cooperation. The indirect trust value was calculated by using density function. It required many resources for calculating the total trust. The proposed scheme implemented the balance between verification rate and communication energy.
Umarani et al. [17] established enhanced trust management system (EBTM) to find internal attack. In the process of communication, the sensor node selects the neighbor node according to the trust information. The state of neighbor node was periodically updated. The recovery process was incorporated to improve network throughput. The scheme not only enhances the cooperation among sensor nodes, but also prolongs the lifetime of the network. However, it does not take into account energy consumption and memory.

Labraoui [18] incorporated risk evaluation into the trust model, and proposed a risk-based trust management system, which could effectively resist malicious attacks. Reputation was evaluated by related observations, and a cumulative evaluation of long-term observation. Risk was assessed based on mutual information. It could detect on-off attack effectively, but not consider more attacks in mobile environment.

Zhu et al. [19] proposed a novel integration scheme, the scheme consists of two sub-schemes, one was the selective data transmission based on time and priority for the gateway, and the other was the priority based sleep scheduling algorithm. These two subschemes solved the problem of data availability and limited node energy respectively. It only addressed issues related to energy consumption, but not specified the ability to resist malicious attacks.

Ganeriwal et al. [20] proposed a scheme for wireless sensor network, which based on reputation distribution (RFSN). The Watchdog and reputation system were two key components in RFSN. The watchdog was used to observe adjacent nodes' behaviors and rate nodes' behaviors as successful or unsuccessful interaction. The model was adopted to maintain nodes' trust. The method adopted an update mechanism to renew trust. Consequently, the credibility of a node is recorded continuously. Obviously, it assumed that each node has enough cooperation with the adjacent neighbor node to make the reputation stable. The effectiveness of the method was verified by simulation experiments. Many scholars have put forward various improvement schemes based on the scheme and achieved good experimental results.

The existing trust management system is complex. Not only the calculation and storage requirements of sensor nodes are high, but also the scope of application is limited. In this paper, based on RFSN, a Poisson distribution-based trust model is proposed. The scheme enables the trust management system simpler and more effective to resist against internal attack.

\section{POISSON DISTRIBUTION-BASED TRUST MODEL}

Based on [21,22] and the relationship between related mathematical distributions, such as Beta distribution, Poisson distribution and so on. We can infer that trust and reputation are represented by the Poisson distribution. The Poisson distribution represents the probability of $\mathrm{k}$ times of cooperation per unit time.

$$
\operatorname{poisson}(k)=e^{-\lambda} \frac{\lambda^{k}}{k !}
$$

Where k represents the number of random events per unit time, $\lambda$ represents the average rate of cooperation between nodes per unit time. let $\lambda=n p$, where $n=a+b$, p represents the probability of successful cooperation. $n$ expresses the total number of interactions 


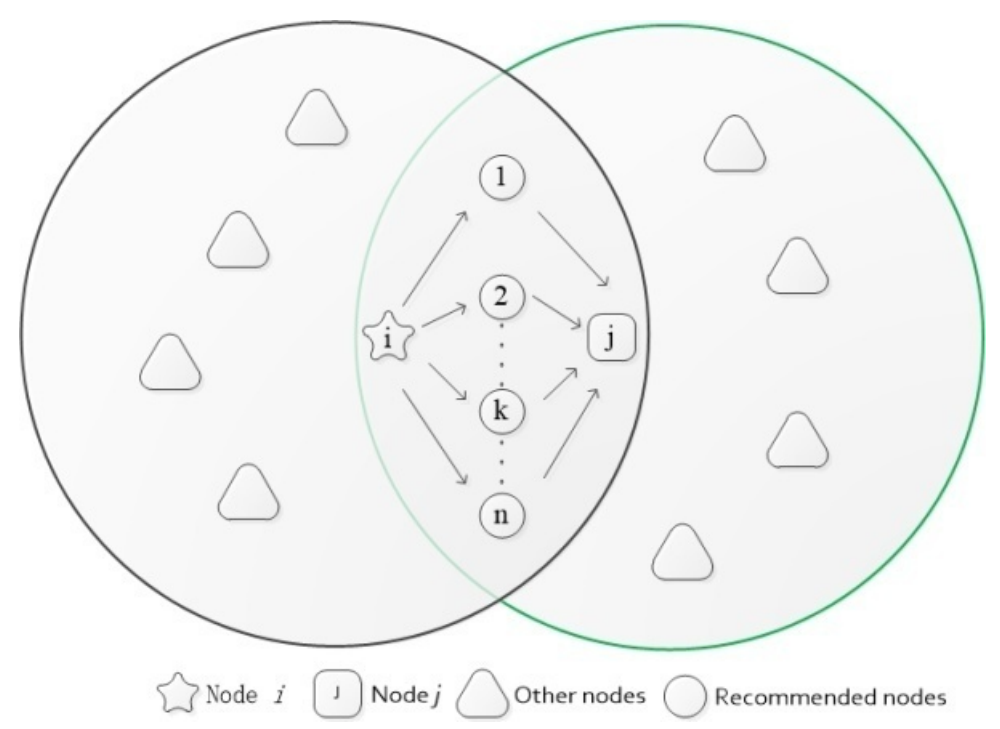

Figure 1: Conveying path of recommendation

between nodes, a represents the number of successful cooperation of the node, and $\mathrm{b}$ represents the number of unsuccessful cooperation of the node. The system can be expressed as follows.

$$
\operatorname{poisson}(k)=e^{-(a+b) p} \frac{((a+b) p)^{k}}{k !}
$$

Using the probability of the number of cooperation between nodes to express the reputation of the nodes, the reputation is the probability distribution of $\mathrm{p}$, we can use the maximum point of the function to express the maximum probability of $p$. thus we defined the maximum value of the function as nodes' trust.

\subsection{Initialize trust value}

Typically, in the phase of initializing trust values, all nodes are defined as the same initial trust value. We assume that all nodes are good nodes. For this hypothesis, it does not require initialization time, but encourages malicious nodes to create a new ID and reenter the network in a form of new reputation. Conversely, it can resolve the problem of forgery of new IDs, but it takes a lot of time to build trust in the system. Based on the above hypothesis, we propose a neutral method that set the initial trust as 0.5 . In other words, let $a$ is equal to $b$. However, it is difficult to determine the number of interactions of $\mathrm{a}$ or $\mathrm{b}$. Due to the number of interactions between nodes is scarce, the trust value is not accurate enough. Contrarily, it not only increases the weight of the historical trust information, affects the evaluation of trust, but also increases the convergence time of the trust value and affects the stability of the network. In general, the selection of reasonable value is significant for $a$ or $b$.

\subsection{Reputation update}

Assume that the reputation indicator of node $i$ and node $j$ is founded between nodes. Cooperation between node $i$ and node $j$ requires $(m+n)$ times, where $m$ indicates the number of successful cooperation and $n$ expresses the number of Unsuccessful cooperation.
Hence, the latest reputation of the node $j$ can be defined as follows.

$$
R_{i j}^{n e w}=\frac{((a+m+b+n) p)^{a+m}}{(a+m) !} e^{-(a+m+n+b)(a+m) p}
$$

\subsection{Indirect observation}

When a node is deemed non-determinacy, the suggestions from third parties need to be required. The assessing node i obtains the recommendation about node $\mathrm{j}$ by adjacent nodes $\mathrm{k}$, marked as $N_{k}$. Node i has a prior distribution $\left(a_{i k}, b_{i k}\right)$ about common neighbor nodes $\mathrm{k}$ already. Node i sends a inquiry message toward its adjacent nodes and the common adjacent nodes held by node $\mathrm{i}$ and node $\mathrm{j}$ responses operation histories with $\left(a_{k j}, b_{k j}\right)$. Accordingly, the trust level of between node $\mathrm{i}$ and node $\mathrm{j}$ can be expressed as $\left(a_{j}, b_{j}\right)$. The working mechanism can be illustrated under the following Fig.1. The circles express the communication range of node $i$ and node jrespectively.

Assume the recommendation offered by the common neighbor nodes $\mathrm{k}$ is $R_{i j}^{k}$. Given $\left(a_{i k}, b_{i k}\right)$ and $\left(a_{i k}, b_{i k}\right)$, the recommendation trust value is calculated as follows, where $\left(R a_{i j}^{k}, R b_{i j}^{k}\right)$ is the recommendation interaction record.

$$
\begin{gathered}
R a_{i j}^{k}=a_{j}+\frac{a_{i k}}{a_{i k}+b_{i k}} \cdot a_{k j} \\
R b_{i j}^{k}=b_{j}+\frac{a_{i k}}{a_{i k}+b_{i k}} \cdot b_{k j} \\
R_{i j}^{k}=\frac{R a_{i j}^{k}}{R a_{i j}^{k}+R b_{i j}^{k}}
\end{gathered}
$$

According to the information i have, not every recommendation is reliable and trustless feedbacks cause error outcome. Apparently, in indirect trust calculation, it is very meaningful to estimate the credibility of the referees accurately. Only the suggestions from 


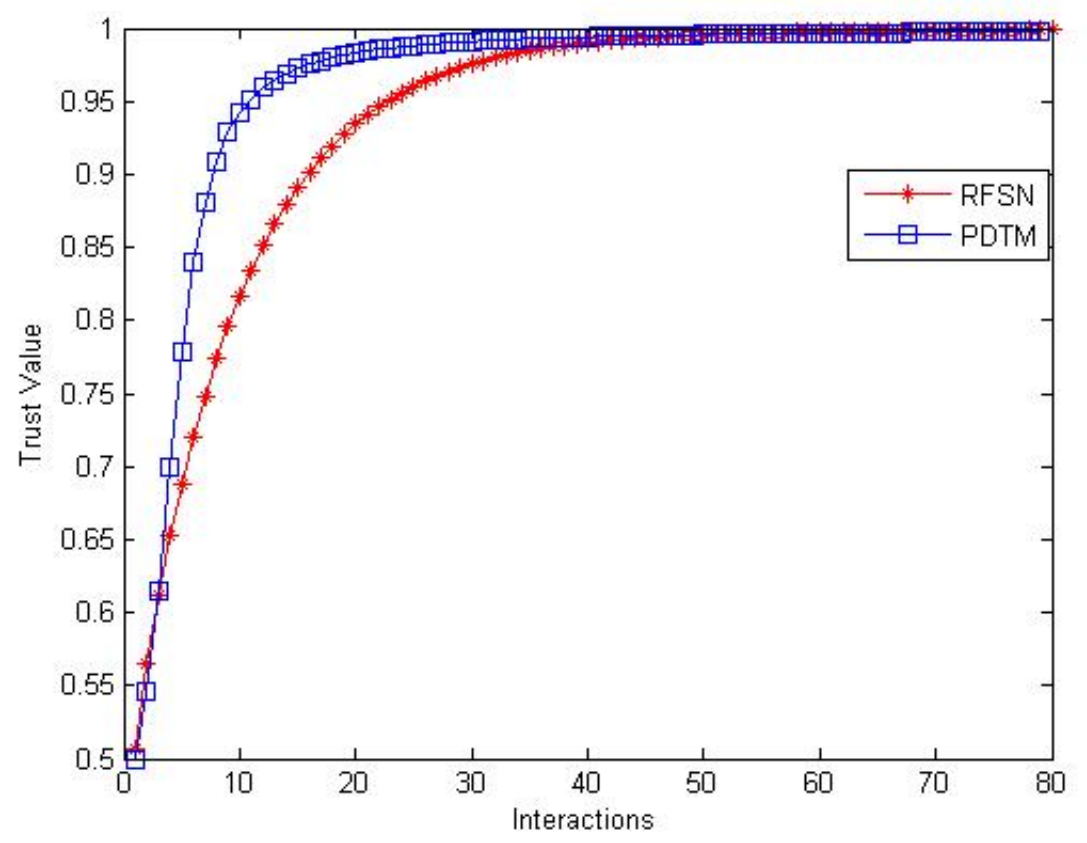

Figure 2: The overall trust of normal nodes

dependable nodes are accepted. The trust of nodeitoward recommender k, symbolized as.

$$
T_{i k}=\frac{a_{i k}}{a_{i k}+b_{i k}}
$$

\section{SIMULATION AND ANALYSES}

In this section, we will assess the security of the method. In the course of performance, PDTM model and typically RFSN model are compared. The experimental results indicate that PDTM can not only reasonably evaluate trust, but also defense malicious attacks.

Suppose that the signal channel is perfect, and normally transmit packers in ideal condition. In the initial phase, we define 0.5 as initial trust. That is aequals $b$. The simulation conditions are as followings: We let the weight is 0.9 , and set the number of adjacent nodes is 2 , the trust factor value is 0.9 , node $i$ assesses neighbor node $\mathrm{j}$, then it is renewed for each interaction.

Assume node $\mathrm{i}$ and $\mathrm{j}$ are reliable, the adjacent nodes is also reliable. Assume that the trust value between node $i$ and node $j$ is $(5,5)$. Suppose the trust of the neighbor nodes to node $j$ as $(5$, 5). Assume that the trust value between node $i$ and its neighbor nodes are $(35,5)$. Finally, we compare PDTM with RFSN in trust evaluation. This simulation results are shown in Fig.2.

Based on the above experimental results, when RFSN and PDTM are adopted to evaluate the reliable nodes, the trust degree of the reliable nodes improves gradually and the performance of PDTM and RFSN are good. The trust of reliable nodes is gradually increasing and the performance of RFSN and PDTM are both good. Relatively, PDTM is better than RFSN due to the fast growth of the trust curve indicates that the initialization time is short. It can save more energy. Briefly speaking, on the basis of the above experimental study indicates that the PDTM has a powerful ability of trust assessment and attack defense.

\section{CONCLUSION}

The interaction state between sensor nodes is a random probability event. Compared with other mathematical distributions, the Poisson distribution is more suitable to express the interaction behavior between nodes.

In PDTM, we adapt the Poisson to represent the nodes' reputation, and then compute trust. What is more, by assigning weights in according to the confidence of the recommenders, the defects caused by subjective weights are avoided. The experimental results demonstrate that, contrasted with RFSN, PDTM is more useful in evaluating trust. Due to terminals in WoTs have constraint in resources, we would like to concentrate on the application of the trust management system and seek for a better way to balance between energy and security. In future work, we will simulate the performance of the method under various scenarios and introduce it in real-world applications.

\section{ACKNOWLEDGMENTS}

This work is partially supported by the National Natural Science Foundation of China (No. 51874300), the National Natural Science Foundation of China and Shanxi Provincial People's Government Jointly Funded Project of China for Coal Base and Low Carbon (No. U1510115). 


\section{REFERENCES}

[1] C.-H. Chen, F. Song, F.-J. Hwang, L. Wu, A Probability Density Function Generator Based on Neural Networks, Physica A: Statistical Mechanics and its Applications, Vol. 541, pp. 123344, 2019.

[2] W. Fang, W. Zhang, W. Chen, Y. Liu, C. Tang. TMSRS: trust management-based secure routing scheme in industrial wireless sensor network with fog computing. Wireless Networks (2020), 26(5): 3169-3182. https://doi.org/10.1007/s11276-01902129-w

[3] C.-H. Chen, A Cell Probe-based Method for Vehicle Speed Estimation, IEICE Transactions on Fundamentals of Electronics, Communications and Computer Sciences, vol. E103-A, no. 1, pp. 265-267, January 2020.

[4] W. Fang, M. Xu, C. Zhu, W. Han, W. Zhang, J. J. P. C. Rodrigues, FETMS: Fast and Efficient Trust Management Scheme for Information-Centric Networking in Internet of Things. IEEE Access. 2019, 7(1): 13476-13485.

[5] W. Fang, W. Zhang, W. Chen, T. Pan, Y. Ni and Y. Yang. Trust-based Attack and Defense in Wireless Sensor Networks: A Survey. Wireless Communications and Mobile Computing. Volume 2020, Article ID 2643546, 20 pages, https://doi.org/10. $1155 / 2020 / 2643546$

[6] C.-H. Chen, F.-J. Hwang, H.-Y. Kung, Travel Time Prediction System Based on Data Clustering for Waste Collection Vehicles, IEICE Transactions on Information and Systems, vol. E102-D, no. 7, pp.1374-1383, July 2019.

[7] W. Fang, N. Cui, W. Chen, W. Zhang, Y. Chen. A Trust-based Security System for Data Collecting in Smart City. IEEE Transactions on Industrial Informatics, doi 10.1109/TII.2020.3006137

[8] C.-H. Chen, An Arrival Time Prediction Method for Bus System, IEEE Internet of Things Journal, vol. 5, no. 5, pp. 4231-4232, October 2018.

[9] A. A. Anasane and R. A. Satao, A Survey on Various Multipath Routing Protocols in Wireless Sensor Networks. Procedia Comput. Sci., vol. 79, no. 20, pp. 610-615, 2016.

[10] C. Zhu, X. Li, V. C. M. Leung, L. T. Yang, E. C.-H. Ngai, and L. Shu, Towards Pricing for Sensor-Cloud. IEEE Trans. Cloud Comput., vol. XX, no. XX, pp. 1-1, 2017.

[11] C. Zhu, V. C. M. Leung, L. T. Yang, and L. Shu, Collaborative Location-Based Sleep Scheduling for Wireless Sensor Networks Integratedwith Mobile Cloud
Computing. IEEE Trans. Comput., vol. 64, no. 7, pp. 1844-1856, 2015

[12] C. Zhu, Z. Sheng, V. C. M. Leung, L. Shu, and L. T. Yang, Toward offering more useful data reliably to mobile cloud from wireless sensor network. IEEE Trans. Emerg. Top. Comput., vol. 3, no. 1, pp. 84-94, 2015.

[13] W. Fang, W. Zhang, Y. Yang, Y. Liu, and W. Chen, A resilient trust management scheme for defending against reputation time-varying attacks based on BETA distribution. Sci. China (Information Sci., vol. 60, no. 4, p. 40305, 2017.

[14] H. Xia, Z. P. Jia, and E. H. M. Sha, Research of trust model based on fuzzy theory in mobile ad hoc networks. IET Inf. Secur., vol. 8, no. 2, pp. 88-103, 2014.

[15] C. Zhu, H. Nicanfar, V. C. M. Leung, and L. T. Yang, An authenticated trust and reputation calculation and management system for cloud and sensor networks integration. IEEE Trans. Inf. Forensics Secur., vol. 10, no. 1, pp. 118-131, 2015.

[16] W. Na and L. DongQian, Trust model based on changeable sampling frequency for wireless sensor network. in 2016 IEEE/ACIS 15th International Conference on Computer and Information Science (ICIS), 2016, pp. 1-4.

[17] V. Umarani, Enhanced Beta Trust Model in Wireless Sensor Networks. in Int. Conf. Inf. Commun. Embed. Syst, 2016, no. Icices, pp. 1-5.

[18] N. Labraoui, A Reliable Trust Management Scheme in Wireless Sensor Networks. in IEEE International Symposium on Programming and Systems (ISPS), 2015, pp. $1-6$.

[19] C. Zhu, X. Li, V. C. M. Leung, L. T. Yang, C. H. Ngai, and L. Shu, Towards Pricing for Sensor-Cloud. IEEE Trans. Cloud Comput., vol. PP, no. 99, p. 1, 2017.

[20] S. Ganeriwal, L. K. Balzano, and M. B. Srivastava, Reputation-based framework for high integrity sensor networks. in ACM Transactions on Sensor Networks, 2008, vol. 4, no. 3, pp. 66-77.

[21] W. Fang, C. Zhang, Z. Shi, Q. Zhao, L. Shan. BTRES: Beta-based Trust and Reputation Evaluation System for Wireless Sensor Networks. Journal of Network and Computer Applications. vol.59, no.1, 2016, pp: 88-94

[22] R. Feng, Q. Liu, Q. Liu, and N. Yu, A credible Bayesian-based trust management scheme for wireless sensor networks. Int. J. Distrib. Sens. Networks, 2015,(201511-1), vol. 2015, no. 2, p. 10, 2015.

[23] D. Hongjun, J. Zhiping, and D. Xiaona, An Entropy-based Trust Modeling and Evaluation for Wireless Sensor Networks. In Proceedings of 2008 International Conference on Embedded Software and Systems. 2008, pp. 27-34. 